\title{
The Nonrelativistic Ground State Energy Spectra of Potential Counting Coulomb and Quad- ratic Terms in Non-commutative Two Dimensional Real Spaces and Phases
}

\author{
Abdelmadjid Maireche \\ Laboratory of Physics and Chemical Materials, Physics Department, \\ Sciences faculty, University of M'sila, M'sila, Algeria
}

(Received 15 December 2015; revised manuscript received 02 March 2016; published online 15 March 2016)

\begin{abstract}
A novel theoretical study for the exact solvability of nonrelativistic quantum spectrum systems for potential containing coulomb and quadratic terms is discussed used both Boopp's shift method and standard perturbation theory in both noncommutativity two dimensional real space and phase (NC-2D: RSP), it has been observed that the exact corrections for the ground states spectrum of studied potential was depended on two infinitesimals parameters $\theta$ and $\bar{\theta}$ which plays an opposite rolls, and we have also constructed the corresponding noncommutative anisotropic Hamiltonian.
\end{abstract}

Keywords: Potential containing coulomb and quadratic terms, Noncommutative space and phase, Boopp's shift method.

\section{INTRODUCTION}

It is well known that the exact energy spectrum, Hamiltonian operators obtained by analytic solutions of the wave equations and corresponding of three fundamental dynamical equations of Schrödinger (SE), KleinGordon and Dirac in the case of (nonrelativistic and relativistic), in commutative and noncommutative spacesphases at two and three dimensional spaces and phases, are possible for some central and non-central potentials [1-45]. Recently new mathematical formulations known by general star product between two arbitrary functions $f(x)$ and $g(x)$ in the first order of two antisymmetric parameters $\left(\theta, \bar{\theta}_{i j}\right)$ can modified the original postulates of quantum mechanics and gives the new commuta$\operatorname{tors}\left[\hat{x}_{\mu}, \hat{x}_{v}\right]_{*}$ and $\left[\hat{p}_{\mu}, \hat{p}_{v}\right]_{*}$, which are playing fundamental rolls' in the non-commutativity geometry of space and phase $(c=\hbar=1)$ [28-45]:

$$
\begin{aligned}
& f(x){ }^{*} g(x)=f(x) g(x)- \\
& -\frac{i}{2} \theta^{\mu v} \partial_{\mu}^{x} f(x) \partial_{v}^{x} g(x)-\frac{i}{2} \bar{\theta}^{\mu v} \partial_{\mu}^{p} f(x) \partial_{v}^{p} g(x) \\
& {\left[\hat{x}_{\mu}, \hat{x}_{v}\right]_{*}=i \theta_{i j} \text { and }\left[\hat{p}_{\mu}, \hat{p}_{v}\right]_{*}=i \bar{\theta}_{i j}}
\end{aligned}
$$

Here, the two parameters $(\theta, \bar{\theta})$ are equal $\left(\theta^{12}, \bar{\theta}^{12}\right)$, respectively. In present work, a Boopp's shift method can be used, instead of solving any quantum systems by using directly star product procedure:

$$
\left[\hat{x}_{\mu}, \hat{x}_{v}\right]=i \theta_{i j} \text { and }\left[\hat{p}_{\mu}, \hat{p}_{v}\right]=i \bar{\theta}_{i j}
$$

The new four operators $\hat{x}, \hat{y}, \hat{p}_{x}$ and $\hat{p}_{y}$ are determined from the relations in (NC-2D: RSP) [28-45]:

$$
\begin{aligned}
& \hat{x}=x-\frac{\theta}{2} p_{y}, \quad \hat{y}=y+\frac{\theta}{2} p_{x} \\
& \hat{p}_{x}=p_{x}+\frac{\bar{\theta}}{2} y \text { and } \hat{p}_{y}=p_{x}-\frac{\bar{\theta}}{2} x
\end{aligned}
$$

We can prove, that the new two uncertainties $\Delta x \Delta y$ and $\Delta p_{x} \Delta p_{y}$ for noncommutative two dimensional spaces and phases are given by, respectively:

$$
\Delta x \Delta y \approx \theta \text { and } \Delta p_{x} \Delta p_{y} \approx \bar{\theta}
$$

The motivation of present search is to present and study the deformed (SE) with potential containing coulomb and quadratic terms in (NC-2D RSP) to discover the new symmetries and a possibility to obtain another application to this potential in different fields and to complete our study in our work [37], we want to obtain new expressions for modified energy levels. Our work based on the provirus work [30-41]. The rest of this work is organized as follows. In next section, we briefly review the (SE) with potential containing coulomb and quadratic terms in two dimensional spaces. The Section 3 , reserved to derive the deformed Hamiltonians of the (SE) with potential containing coulomb and quadratic terms and by applying both Boopp's shift method and standard perturbation theory we find the quantum spectrum of ground states in (NC-2D: RSP) for studied potential. In next section we resume the global obtained spectrum and corresponding deformed Hamiltonian. Finally, the important found results and the conclusions are discussed in last section.

\section{REVIEW OF POTENTIAL CONTAINING COULOMB AND QUADRATIC TERMS IN ORDINARY TWO DIMENSIONAL SPACE}

The two-dimensional stationary (SE) with potential $V(r)=a r^{2}-\frac{b}{r}$ describing potential containing coulomb and quadratic terms, depending only on the distance $r$ 
from the origin:

$$
\left(-\frac{\Delta}{2 \mu}+a r^{2}-\frac{b}{r}\right) \Psi_{n, l, m}(r, \phi)=E_{n, l} \Psi_{n, l, m}(r, \phi)
$$

Where $\mu$ and $E_{n, l}$ denotes to the reduced mass and the energy respectively, the coefficients $a$ and $b$ are both constants. The Laplacian operator takes the values in polar coordinates:

$$
\Delta=\frac{1}{r} \frac{\partial}{\partial r}\left(r \frac{\partial}{\partial r}\right)+\frac{1}{r^{2}} \frac{\partial^{2}}{\partial \phi^{2}}
$$

The wave function can be written as [1]:

$$
\Psi_{n, l, m}(r, \phi)=R_{n, l}(r) \exp ( \pm i m \phi)
$$

The eigenstate $R_{n, l}(r)$ for coulomb and quadratic terms potential satisfied the reduced radial differential function [1]:

$$
\begin{aligned}
& \frac{d^{2} R_{n, l}(r)}{d r^{2}}+\frac{1}{r} \frac{d R_{n, l}(r)}{d r}-\frac{l^{2}}{r^{2}} R_{n, l}(r)+ \\
& +2 \mu\left(E_{n, l}-a r^{2}+\frac{b}{r}\right) R_{n, l}(r)=0
\end{aligned}
$$

The eq. (7) accepts a solution for a radial function $R_{n, l}(r)$, as follows [1]:

$$
R_{n, l}(r)=N_{n, l} \exp \left(-\sqrt{\beta} \frac{r^{2}}{2}\right) f_{n, l}(r)
$$

Where $N_{n, l}$ the normalization factor, $\beta$ and $f_{n, l}(r)$ are determined from two equations, respectively [1]:

$$
\left\{\begin{array}{c}
\beta=2 \mu a \\
f_{n, l}(r)=r^{k} \sum_{n=0}^{\infty} a_{n} r^{n}
\end{array}\right.
$$

The factors $a_{n}$ determined from the following relations [1]:

$$
\begin{aligned}
& a_{0} \neq 0 \\
& a_{1}=\frac{2 \mu b a_{0}}{2 l+1} \\
& a_{n}=\frac{2 \sqrt{\beta}\left(n_{r}+l-1\right) a_{n-2}-2 \mu b a_{n-1}}{\left(n_{l}+l\right)^{2}-l^{2}}
\end{aligned}
$$

In two dimensional spaces, the energy $E_{n_{r}, l}$ depended by the factors $a_{n}$ from the following projection [1]:

$$
E_{n_{r}, l}=\sqrt{\frac{2}{\mu} a}\left(n_{r}+l-1\right)-b \frac{a_{n_{r}-1}}{a_{n_{r}-2}}
$$

The radial part and the energy of (ordinary Hydrogen atom) counting quadratic term potential for the ground state, respectively [1]:

$$
R(r)=N_{2,1} \exp \left(-\sqrt{\beta} \frac{r^{2}}{2}\right) r^{l}\left(1-\frac{\gamma}{2 l+1} r\right)
$$

and

$$
E_{0, l}=\sqrt{\frac{2}{\mu} a}(l+1)+\frac{b}{2 l+1}
$$

where $N_{2,1}$ denote to the normalization constant.

\section{TWO DIMENSIONAL NONCOMMUTATIVE SPACE AND PHASE FOR POTENTIAL CON- TAINING COULOMB AND QUADRATIC TERMS}

Know, we present some fundamental principles of modified Schrödinger equation in (NC-2D: RSP); applying the important 4-steps on the ordinary quantum (SE) [31-42]:

- we replace ordinary two dimensional Hamiltonian operators $\hat{H}\left(p_{i}, x_{i}\right)$ by noncommutative new Hamilto$\operatorname{nian} \hat{H}\left(\hat{p}_{i}, \hat{x}_{i}\right)$,

- we replace ordinary complex wave function $\Psi(\vec{r})$ by new complex wave function $\hat{\Psi}(\overrightarrow{\vec{r}})$,

- we replace ordinary energy $E_{n, l}$ by noncommutative energy $E_{n c-q c}$,

- the last steps correspond to replace the ordinary old product by new star product.

Which allow us to construct the modified Schrödinger equitation in both (NC-2D: RSP) as:

$$
\hat{H}\left(\hat{p}_{i}, \hat{x}_{i}\right) * \hat{\Psi}(\overrightarrow{\vec{r}})=E_{n c-q c} \hat{\Psi}(\overrightarrow{\vec{r}})
$$

Now, we apply the Boopp's shift method on the above equation to obtain, the reduced Schrödinger equation (without star products):

$$
H\left(\hat{p}_{i}, \hat{x}_{i}\right) \psi(\vec{r})=E_{n c-q c} \psi(\vec{r})
$$

This is a translation of a Schrödinger equation for $\hat{p}_{i}$ and $\hat{x}_{i}$ with the same complex wave function $\psi(\vec{r})$. As a direct result of the eq. (3), the two operators $\hat{r}^{2}$ and $\hat{p}^{2}$ in (NC-2D RSP) can be written as follows [30-41]

$$
\left\{\begin{array}{l}
\hat{r}^{2}=r^{2}-\theta L_{z} \\
\hat{p}^{2}=p^{2}+\bar{\theta} L_{z}
\end{array}\right.
$$

Here $L_{z} \equiv x p_{y}-y p_{x}$.It's important to notice that the rolls of $\theta$ and $\bar{\theta}$ are $\operatorname{inversed}(\theta \approx-\bar{\theta})$. After a straightforward calculation, we can obtain the three important terms:

$$
\begin{aligned}
& a \hat{r}^{2}=a r^{2}-a \theta L_{z} \\
& \frac{b}{\hat{r}}=\frac{b}{r}+\frac{\theta b}{2 r^{3}} L_{z} \\
& \frac{\hat{p}^{2}}{2 m_{0}}=\frac{p^{2}}{2 m_{0}}+\frac{\bar{\theta}}{2 m_{0}} L_{z}
\end{aligned}
$$


Which will be use to determine the deformed potential containing coulomb and quadratic terms $V(\hat{r})$ and the new deformed kinetic term $\frac{\hat{p}^{2}}{2 m_{0}}$, in (NC-2D: RSP), respectively:

$$
\begin{aligned}
& V(\hat{r})=a \hat{r}^{2}-\frac{b}{\hat{r}} \\
& \frac{\hat{p}^{2}}{2 m_{0}}=-\frac{1}{2 m_{0}}\left(\frac{1}{r} \frac{\partial}{\partial r}\left(r \frac{\partial}{\partial r}\right)+\frac{1}{r^{2}} \frac{\partial^{2}}{\partial \phi^{2}}\right)+\frac{\bar{\theta}}{2 m_{0}} L_{z}
\end{aligned}
$$

It is well known, that the angular momentum is perpendicular to area of motion in two dimensional spaces, then the obtained result is naturally, which allow us to obtaining the global potential operator $H(\hat{r})$ for potential containing coulomb and quadratic terms in both (NC-2D: RSP) as:

$$
H(\hat{r})=a r^{2}-\frac{b}{r}+\left(\theta\left(\frac{b}{2 r^{3}}-a\right)+\frac{\bar{\theta}}{2 m_{0}}\right) L_{z}
$$

It's clearly, that the two first terms are given the ordinary potential containing coulomb and quadratic terms in two dimensional space while the rest terms are proportional's with two infinitesimals parameters $(\theta$ and $\bar{\theta}$ ) and then gives the terms of perturbation $H(r)$ for potential containing coulomb and quadratic terms in (NC-2D RSP) as:

$$
H(r)=\left(\theta\left(\frac{b}{2 r^{3}}-a\right)+\frac{\bar{\theta}}{2 m_{0}}\right) L_{z}
$$

This can be writing to the equivalent form:

$$
H(r)=\left(\theta\left(\frac{b}{2 r^{3}}-a\right)+\frac{\bar{\theta}}{2 m_{0}}\right) \vec{S} \vec{L}
$$

We orient the spin to the $(\mathrm{Oz})$ which appear parallel with $L_{z}$, which allow us to write, the perturbative term $H(r)$ as follows:

$$
H(r)=\left(\theta\left(\frac{b}{2 r^{3}}-a\right)+\frac{\bar{\theta}}{2 m_{0}}\right)\left(\overleftrightarrow{J}^{2}-\overleftrightarrow{L}^{2}-\overleftrightarrow{S}^{2}\right)
$$

We have replaced $(\vec{S} \vec{L})$ by $\frac{1}{2}\left(\overleftrightarrow{J}^{2}-\overleftrightarrow{L}^{2}-\overleftrightarrow{S}^{2}\right)$, this operator traduce the coupling between spin and orbital momentum. After profound straightforward calculation, one can show that, the radial function $R_{n l}(r)$ and the angular function $\Phi(\phi)$ are satisfied the following two equations, in (NC-2D: RSP), respectively for potential containing coulomb and quadratic terms:

$$
\begin{aligned}
& \frac{d^{2} R_{n, l}(r)}{d r^{2}}+\frac{1}{r} \frac{d R_{n, l}(r)}{d r}-\frac{l^{2}}{r^{2}} R_{n, l}(r)+ \\
& +2 \mu\left(E_{n c-q c}-a r^{2}+\frac{b}{r}\right) R_{n, l}(r) \\
& -2 \mu\left(\theta\left(\frac{b}{2 r^{3}}-a\right)+\frac{\bar{\theta}}{2 m_{0}}\right) L_{z} R_{n, l}(r)=0
\end{aligned}
$$

and

$$
\frac{d^{2} \Phi_{m}(\phi)}{d \phi^{2}}+m^{2} \Phi_{m}(\phi)=0
$$

The set ( $H, \mathrm{~J}^{2}, \mathrm{~L}^{2}, \mathrm{~S}^{2}$ and $J_{z}$ ) forms a complete of conserved physics quantities and the eigen-values of the spin orbital coupling operator are $k_{ \pm} \equiv \frac{1}{2}\left\{\left(l \pm \frac{1}{2}\right)\left(l \pm \frac{1}{2}+1\right)+l(l+1)-\frac{3}{4}\right\}$ corresponding: $j=l+\frac{1}{2}$ (spin up) and $j=l-\frac{1}{2}$ (spin down), respectively [30-41], then, one can form a diagonal $(2 \times 2)$ matrix, with non null elements are $\left(H_{s o}\right)_{11}$ and $\left(H_{s o}\right)_{22}$ for potential containing coulomb and quadratic terms in (NC2D RSP) as:

$$
\begin{gathered}
\left(H_{s o}\right)_{11}=k_{+}\left(\theta\left(\frac{b}{2 r^{3}}-a\right)+\frac{\bar{\theta}}{2 m_{0}}\right) \text { if } j=l+\frac{1}{2} \Rightarrow \text { spin up } \\
\left(H_{s o}\right)_{22}=k_{-}\left(\theta\left(\frac{b}{2 r^{3}}-a\right)+\frac{\bar{\theta}}{2 m_{0}}\right) \text { if } j=l-\frac{1}{2} \Rightarrow \text { spin down }
\end{gathered}
$$

\section{THE NONCOMMUTATIVE SPECTRA FOR (ORDINARY HYDROGEN ATOM) COUNTING QUADRATIC TERM POTENTIAL IN TWO DIMENSIONAL NONCOMMUTATIVE SPACE AND PHASE}

The exact values for energies states $E_{N U-C Q}$ and $E_{N D-C Q}$ of an electron with spin up and spin down, corresponding the two operators $\left(H_{U}\right)_{11}$ and $\left(H_{D}\right)_{22}$ are determined to be, respectively

$$
\begin{aligned}
& E_{N U-C Q}=E_{0}+E_{U-C Q} \\
& E_{N D-C Q}=E_{0}+E_{D-C Q}
\end{aligned}
$$

where $E_{U-C Q}$ and $E_{D-C Q}$ are the modifications to the energy levels, associated with spin up and spin down at first order of two infinitesimals parameters ( $\theta$ and $\bar{\theta}$ ) and by applying the perturbation theory, $E_{U-C Q}$ and $E_{D-C Q}$ became, respectively

$$
\begin{gathered}
\frac{E_{U-C Q}}{2 \prod \alpha^{\prime} L\left(l, j=l+\frac{1}{2}, s\right)}= \\
=\int \Psi^{(p) *}(\vec{r})\left\{\theta\left(\frac{b}{2 r^{3}}-a\right)+\frac{\bar{\theta}}{2 m_{0}}\right\} \Psi^{(p)}(\vec{r}) r d r \\
\frac{E_{D-C Q}}{2 \prod \alpha^{\prime} L^{\prime}\left(l, j=l-\frac{1}{2}, s\right)}= \\
=\int \Psi^{(p) *}(\vec{r})\left\{\theta\left(\frac{b}{2 r^{3}}-a\right)+\frac{\bar{\theta}}{2 m_{0}}\right\} \Psi^{(p)}(\vec{r}) r d r .
\end{gathered}
$$

The non-commutative modifications of the energy levels, associated with spin up and spin down, in the first order of corresponding ( $E_{0 U}$ and $E_{0 D}$ ) are determined using Esq. (11), (22) and (29) to obtain 


$$
\begin{aligned}
& \frac{E_{U-C Q}}{2 \prod k_{+}}=\int \exp \left(-\sqrt{\beta} r^{2}\right) r^{2 l+1} \times \\
& \times\left(1+\left(\frac{\gamma}{2 l+1}\right)^{2} r^{2}-\frac{2 \gamma}{2 l+1} r\right)\left\{\begin{array}{l}
\theta\left(\frac{b}{2 r^{3}}-a\right) \\
+\frac{\bar{\theta}}{2 m_{0}}
\end{array}\right\} d r \\
& \frac{E_{D-C Q}}{2 \prod k_{-}}=\int \exp \left(-\sqrt{\beta} r^{2}\right) r^{2 l+1} \times \\
& \times\left(1+\left(\frac{\gamma}{2 l+1}\right)^{2} r^{2}-\frac{2 \gamma}{2 l+1} r\right)\left\{\begin{array}{l}
\theta\left(\frac{b}{2 r^{3}}-a\right) \\
+\frac{\bar{\theta}}{2 m_{0}}
\end{array}\right\} d r
\end{aligned}
$$

A direct simplification gives:

$$
\begin{aligned}
& E_{U-C Q}=2 \prod k_{+}\left(\theta \sum_{i=1}^{6} T_{i}+\frac{\bar{\theta}}{2 m_{0}} \sum_{i=7}^{9} T_{i}\right) \\
& E_{D-C Q}=2 \prod k_{-}\left(\theta \sum_{i=1}^{6} T_{i}+\frac{\bar{\theta}}{2 m_{0}} \sum_{i=7}^{9} T_{i}\right)
\end{aligned}
$$

Where the notations $T_{i}$ are given by:

$$
\begin{aligned}
& T_{1}=\frac{b}{2} \int_{0}^{+\infty} \exp \left(-\sqrt{\beta} r^{2}\right) r^{2 l-2} d r \\
& T_{2}=-a \int_{0}^{+\infty} \exp \left(-\sqrt{\beta} r^{2}\right) r^{2 l+1} d r \\
& T_{3}=\frac{b}{2}\left(\frac{\gamma}{2 l+2}\right) \int_{0}^{2+\infty} \exp \left(-\sqrt{\beta} r^{2}\right) r^{2 l} d r \\
& T_{4}=-a\left(\frac{\gamma}{2 l+2}\right)^{2} \int_{0}^{+\infty} \exp \left(-\sqrt{\beta} r^{2}\right) r^{2 l+3} d r \\
& T_{5}=-\frac{b}{2} \frac{2 \gamma}{2 l+1} \int_{0}^{+\infty} \exp \left(-\sqrt{\beta} r^{2}\right) r^{2 l-1} d r \\
& T_{6}=-a \frac{2 \gamma}{2 l+1} \int_{0}^{+\infty} \exp \left(-\sqrt{\beta} r^{2}\right) r^{2 l+2} d r
\end{aligned}
$$

and

$$
\begin{aligned}
& T_{7}=\frac{b}{2} \int_{0}^{+\infty} \exp \left(-\sqrt{\beta} r^{2}\right) r^{2 l+1} d r \\
& T_{8}=\left(\frac{\gamma}{2 l+2}\right)^{2} \int_{0}^{+\infty} \exp \left(-\sqrt{\beta} r^{2}\right) r^{2 l+3} d r \\
& T_{9}=-\frac{2 \gamma}{2 l+1} \int_{0}^{+\infty} \exp \left(-\sqrt{\beta} r^{2}\right) r^{2 l+2} d r
\end{aligned}
$$

We use the following form of special integral [46]

$$
\int_{0}^{+\infty} x^{m} \exp \left(-\alpha x^{n}\right) d x=-\frac{\Gamma\left(\frac{m+1}{n}, \alpha x^{n}\right)}{n \alpha^{\frac{m+1}{n}}}
$$

Where $\Gamma\left(\frac{m+1}{n}, \alpha x^{n}\right)$ is the incomplete gamma function, then we obtains the following results

$$
\begin{aligned}
& T_{1}=-\frac{b}{2} \frac{\Gamma\left(\frac{2 l-1}{2}, \beta r^{2}\right)}{2 \beta^{\frac{2 l-1}{4}}}, \\
& T_{2}=a \frac{\Gamma\left(\frac{2 l+2}{2}, \beta r^{2}\right)}{2 \beta^{\frac{2 l+2}{4}}} \\
& T_{3}=-\frac{b}{2}\left(\frac{\gamma}{2 l+2}\right)^{2} \frac{\Gamma\left(\frac{2 l+1}{2}, \beta r^{2}\right)}{2 \beta^{\frac{2 l+1}{4}}}
\end{aligned}
$$

and

$$
\begin{aligned}
& T_{7}=\frac{b}{2} \frac{\Gamma\left(\frac{2 l++}{2}, \beta r^{2}\right)}{2 \beta^{\frac{2 l+2}{4}}}, \\
& T_{8}=\left(\frac{\gamma}{2 l+2}\right)^{2} \frac{\Gamma\left(\frac{2 l+4}{2}, \beta r^{2}\right)}{2 \beta^{\frac{2 l-1}{4}}} \\
& T_{9}=-\frac{2 \gamma}{2 l+1} \frac{\Gamma\left(\frac{2 l+3}{2}, \beta r^{2}\right)}{2 \beta^{\frac{2 l+3}{4}}}
\end{aligned}
$$

and,

$$
\begin{aligned}
& T_{4}=a\left(\frac{\gamma}{2 l+2}\right)^{2} \frac{\Gamma\left(\frac{2 l+4}{2}, \beta r^{2}\right)}{2 \beta^{\frac{2 l+4}{4}}} \\
& T_{5}=\frac{b}{2} \frac{2 \gamma}{2 l+1} \frac{\Gamma\left(\frac{2 l}{2}, \beta r^{2}\right)}{2 \beta^{\frac{2 l}{4}}} \\
& T_{6}=a \frac{2 \gamma}{2 l+1} \frac{\Gamma\left(\frac{2 l+3}{2}, \beta r^{2}\right)}{2 \beta^{\frac{2 l+3}{4}}}
\end{aligned}
$$

Which allow us to obtaining the exact energy of ground state in (NC-2D: RSP) spaces and phases for potential of (ordinary Hydrogen atom) counting quadratic term associated with spin up and spin down in the first order perturbation of $\theta$ and $\bar{\theta}$ as follows

$$
\begin{aligned}
& E_{U-C Q}=2 \prod k_{+}\left(\theta T_{2 s}+\frac{\bar{\theta}}{2 m_{0}} T_{2 p}\right) \\
& E_{D-C Q}=2 \prod k_{-}\left(\theta T_{2 s}+\frac{\bar{\theta}}{2 m_{0}} T_{2 p}\right)
\end{aligned}
$$

Where

$$
T_{2 s}=\sum_{i=1}^{6} T_{i} \text { and } \quad T_{2 p}=\sum_{i=7}^{9} T_{i}
$$

We conclude, from Eqs. (13.2), (33.1) and (33.2) the total energy of electron with two polarizations spin up and down $E_{N U-C Q}$ and $E_{N D-C Q}$ for potential containing coulomb and quadratic terms in (NC-2D: RSP) produced by the effect of spin-orbital interaction as:

$$
\begin{aligned}
& E_{N U-C Q}=\sqrt{\frac{2}{\mu} a}(l+1)+\frac{b}{2 l+1}+ \\
& +2 \prod k_{+}\left(\theta T_{2 s}+\frac{\bar{\theta}}{2 m_{0}} T_{2 p}\right) \\
& E_{N D-C Q}=\sqrt{\frac{2}{\mu} a}(l+1)+\frac{b}{2 l+1}+ \\
& +2 \prod k_{-}\left(\theta T_{2 s}+\frac{\bar{\theta}}{2 m_{0}} T_{2 p}\right)
\end{aligned}
$$

On another hand it's possible to writing the corresponding non-commutative Hamiltonian $H_{N C 1}$ as fol- 
lows:

$$
H_{N C 1}=H+H_{s o .}
$$

Where $H$ and $H_{\text {so. }}$ are determined from, the following relation, respectively:

$$
\begin{aligned}
& H=\left(-\frac{1}{2 m_{0}}\left(\frac{1}{r} \frac{\partial}{\partial r}\left(r \frac{\partial}{\partial r}\right)+\frac{1}{r^{2}} \frac{\partial^{2}}{\partial \phi^{2}}\right)+a r^{2}-\frac{b}{r}\right)\left(\begin{array}{ll}
1 & 0 \\
0 & 1
\end{array}\right) \\
& H_{s o}=\left(\theta\left(\frac{b}{2 r^{3}}-a\right)+\frac{\bar{\theta}}{2 m_{0}}\right)\left(\begin{array}{cc}
k_{+} & 0 \\
0 & -k_{-}
\end{array}\right)
\end{aligned}
$$

Furthermore, if we apply the three-following steps:

$$
\begin{aligned}
& \left(\theta\left(\frac{b}{2 r^{3}}-a\right)+\frac{\bar{\theta}}{2 m_{0}}\right) L_{z} \rightarrow\left(\chi\left(\frac{b}{2 r^{3}}-a\right)+\frac{\bar{\sigma}}{2 m_{0}}\right) \vec{B} \vec{L} \\
& \theta \rightarrow \chi B \text { and } \bar{\theta} \rightarrow \bar{\sigma} B
\end{aligned}
$$

And we ordinate the magnetic field $\overleftrightarrow{B}=B \vec{k}$ to (oz) axis, $\chi$ and $\bar{\sigma}$ are two infinitesimal real proportional's constants, the magnetic moment $\vec{\mu} \equiv 1 / 2$ and $(-\vec{S} \vec{B})$ denote to the ordinary Hamiltonian of Zeeman Effect, we obtains the modified new modified Hamiltonian $H_{m}$ for potential containing coulomb and quadratic terms in (NC-2D:RSP) as:

$$
H_{m}=\left(\chi\left(\frac{b}{2 r^{3}}-a\right)+\frac{\bar{\sigma}}{2 m_{0}}\right)(\vec{B} \vec{J}-\vec{S} \vec{B})
$$

The above operator represents modified fundamentals interactions between spin and external uniform magnetic field (containing ordinary Zeeman Effect).To obtain the exact non-commutative magnetic modifications of energy $E_{\text {mag-0 }}$ for potential containing coulomb and quadratic terms corresponding ground state in (NC-2D: RSP), its sufficient to replace the three parameters: $k_{+}, \theta$ and $\bar{\theta}$ in the eq.(35.1) by the following new parameters: $\mathrm{m}, \chi$ and $\bar{\sigma}$, respectively:

$$
E_{\text {mag- } 0}=2 \prod m B\left(\chi T_{2 s}+\frac{\bar{\sigma}}{2 m_{0}} T_{2 p}\right)
$$

With $-l \leq m \leq+l$, which allow us to fixing $(2 l+1)$ values for discreet number $m$.

\section{DISCUSSIONS THE OBTAINED RESULTS}

We want to construct the complete NC Hamiltonian from Eqs. (37) and (39), which allow us to deduce the following diagonal matrix $H_{N C-2 C Q}$ for the coulomb and quadratic terms potential in (NC-2D: RSP) as

$$
H_{N C-2 C Q}=\left(\begin{array}{cc}
\left(H_{N C-2 C Q}\right)_{11} & 0 \\
0 & \left(H_{N C-2 C Q}\right)_{22}
\end{array}\right)
$$

Where the two elements $\left(H_{N C-C Q}\right)_{11}$ and $\left(H_{N C-C Q}\right)_{22}$ are determined by the two explicitly physical form

$$
\begin{aligned}
& \left(H_{N C-2 C Q}\right)_{11}=-\frac{1}{2 m_{0}}\left(\frac{1}{r} \frac{\partial}{\partial r}\left(r \frac{\partial}{\partial r}\right)+\frac{1}{r^{2}} \frac{\partial^{2}}{\partial \phi^{2}}\right) \\
& +a r^{2}-\frac{b}{r}+k_{+}\left(\theta\left(\frac{b}{2 r^{3}}-a\right)+\frac{\bar{\theta}}{2 m_{0}}\right) \\
& +\left(\chi\left(\frac{b}{2 r^{3}}-a\right)+\frac{\bar{\sigma}}{2 m_{0}}\right) \vec{B} \vec{L}
\end{aligned}
$$

and

$$
\begin{aligned}
& \left(H_{N C-2 C Q}\right)_{22}=-\frac{1}{2 m_{0}}\left(\frac{1}{r} \frac{\partial}{\partial r}\left(r \frac{\partial}{\partial r}\right)+\frac{1}{r^{2}} \frac{\partial^{2}}{\partial \phi^{2}}\right) \\
& +a r^{2}-\frac{b}{r}+k_{-}\left(\theta\left(\frac{b}{2 r^{3}}-a\right)+\frac{\bar{\theta}}{2 m_{0}}\right)
\end{aligned}
$$

Finally, the modified spectrum for ground states $E_{N U-G C Q}$ and $E_{N D-G C Q}$ corresponding fermionic particle with spin up and down produced by modified potential containing coulomb and quadratic terms can be deduced from the partial results (35.1), (35.2) and (40):

$$
\begin{aligned}
& E_{N U-G C Q}=\sqrt{\frac{2}{\mu} a}(l+1)+\frac{b}{2 l+1}+ \\
& +2 \prod k_{+}\left(\theta T_{2 s}+\frac{\bar{\theta}}{2 m_{0}} T_{2 p}\right)+ \\
& +2 \prod m B\left(\chi T_{2 s}+\frac{\bar{\sigma}}{2 m_{0}} T_{2 p}\right)
\end{aligned}
$$

and

$$
\begin{aligned}
& E_{N D-G C Q}=\sqrt{\frac{2}{\mu} a}(l+1)+\frac{b}{2 l+1}+ \\
& +2 \prod k_{-}\left(\theta T_{2 s}+\frac{\bar{\theta}}{2 m_{0}} T_{2 p}\right)+ \\
& +2 \prod m B\left(\chi T_{2 s}+\frac{\bar{\sigma}}{2 m_{0}} T_{2 p}\right)
\end{aligned}
$$

If we consider the $\operatorname{limits}(\theta, \bar{\theta}) \rightarrow(0,0)$, the above spectrum reduces to the ordinary spectrum in two dimensional spaces which obtained from the reference [1].

\section{CONCLUSIONS}

In this work, we have applied both Boopp's shift method and standard perturbation theory to obtain the exact energy spectrum for ground state with coulomb and quadratic terms potential in noncommutative two dimensional real spaces and phase's spaces. We shown that the ordinary ground state in two dimensional spaces changed and replaced by degenerated new states, corresponding two polarized states spin up and spin down as it's observed in ordinary Dirac equation at high energy, thus our study replaced the ordinary nonrelativistic spectrum by a new relativistic spectrum valid at height energies.

\section{ACKNOWLEGMENTSA}

This work was supported with search laboratory of: Physical and material chemical, in university of M'sila, Algeria. 


\section{REFERENCES}

1. H. Hassanabadi, M. Hamzavi, S. Zarrinkamar, A.A. Rajabi, Int. J. Phys. Sci. 6 No 3, 583 (2011).

2. S.K. Bose, Il Nuovo Cimento 109, 1217 (1994).

3. L. Buragohain, S.A.S. Ahmed, Lat. Am. J. Phys. Educ. 4 No 1 (2010).

4. S. A. Raushal, S.A. Ahmed, B.C. Borah, D. Sarma, Eur. Phys. J. D 17, 335 (1994).

5. Sameer M. Ikhdair, Ramazan Sever, CEJP 5 No 4, 516 (2007).

6. M.M. Nieto, Am. J. Phys. 47, 1067 (1979).

7. S.M. Ikhdair, R. Sever, J. Mol. Struc.-Theochem. 806, 155 (2007).

8. A.S. Ahmed, L. Buragohain, Phys. Scr. 80, 025004 (2009).

9. S.K. Bose, Nouvo Cimento B 113, 299 (1996).

10. G.P. Flesses, A. Watt, J. Phys. A: Math. Gen. 14, L315 (1981).

11. M. Ikhdair, R. Sever, Ann. Phys. (Leipzig) 16, 218 (2007).

12. S.H. Dong, Phys. Scripta 64 No 4, 273 (2001).

13. S.H. Dong, Z.-Q. Ma, J. Phys. A 31 No 49, 9855 (1998).

14. S.H. Dong, Int. J. Theoretical Phys. 40 No 2, 559 (2001).

15. Ali Akder, et al., J. Theoretical Appl. Phys. 7, 17 (2013).

16. Sameer M. Ikhdair, Ramazan Sever, Adv. High Energ. Phys. 2013, ID 562959 (2013).

17. Shi-Hai Dong, Guo-Hua San, Foundation. Phys. Lett. 16 No 4, 357 (2003).

18. L. Buragohain, S.A.S. Ahmed, Lat. Am. J. Phys. Educ. 4, No 1, 79 (2010).

19. S.M. Ikhdair, J. Modern Phys. 3 No 2, 170 (2012).

20. H. Hassanabadi, et al., Tur. Phys. J. Plus. 127, 120 (2012).

21. Shi-Hai Dong, Zhoung-Qi Ma, Giampieero Esposito, Fondation. Phys. Lett. 12 No 5, (1999).

22. A. Connes, Non-commutative geometry, $1^{\text {st }}$ Ed. (Academic Press: Paris, France: 1994).

23. H. Snyder, Phys. Rev. 71, 38 (1946).

24. Anselme F. Dossa, Gabriel Y.H. Avossevou, J. Modern Phys. 4, 1400 (2013).
25. D.T. Jacobus, PhD, Department of Physics, Stellenbosch University, South Africa (2010).

26. Anais Smailagic, et al., Phys. Rev. D 65, 107701 (2002).

27. Yang, Zu-Hua, et al., Int. J. Theor. Phys. 49, 644 (2010).

28. Y. Yuan, et al., Chinese Physics C 34 No 5, 543 (2010).

29. Behrouz Mirza, et al., Commun. Theor. Phys. 55, 405 (2011).

30. Abdelmadjid Maireche, Life Sci. J. 11 No 6, 353 (2014).

31. Abdelmadjid Maireche, The African Rev. Phys. 9:0060, 479 (2014).

32. Abdelmadjid Maireche, J. Nano- Electron. Phys. 7 No 2, 02003 (2015)

33. Abdelmadjid Maireche, The African Rev. Phys. 9:0025, 185 (2014).

34. Abdelmadjid Maireche, The African Rev. Phys. 10:0014, 97 (2015).

35. Abdelmadjid Maireche, Int. Lett. Chem., Phys. Astronomy 56, 1 (2015).

36. Abdelmadjid Maireche, Int. Lett. Chem., Phys. Astronomy 60, $11(2015)$

37. Abdelmadjid Maireche, The African Rev. Phys. 10:0025, 177 (2015).

38. Abdelmadjid Maireche, Int. Lett. Chem., Phys. Astronomy 58, 164 (2015).

39. Abdelmadjid Maireche, Med. J. Model. Simul. 4, 60 (2015).

40. Abdelmadjid Maireche, The African Rev. Phys. 9:0025, 479 (2014).

41. Abdelmadjid Maireche, J. Nano- Electron. Phys. 7 No 4, 04021 (2015).

42. A.E.F. Djemei, H. Smail, Commun. Theor. Phys. (Beijinig, China) 41, 837 (2004).

43. Shaohong Cai, Tao Jing, Guangjie Guo, Rukun Zhang, Int. J. Theoretical Phys. 49 No 8, 1699 (2010).

44. Joohan Lee, J. Korean Phys. Soc. 47 No 4, 571 (2005).

45. A. Jahan, Braz. J. Phys. 37 No 4, 144 (2007).

46. I.S. Gradshteyn, I.M. Ryzhik, Table of Integrals, Series and Products, $7^{\text {th }}$ Ed. (Elsevier: 2007). 